\title{
A CASE REPORT OF DEEP FIBROMATOSIS OF SACRAL REGION
}

KEY WORDS:

\section{Dr.}

Padmanabhan

Prabhakar

\section{Dr. Sindhu K*}

\section{Prof R. Kannan}

Assistant Professor , Institute of General Surgery, Madras Medical college and Rajiv Gandhi Government General Hospital, Chennai.

Postgraduate, Institute of General Surgery, Madras Medical college and Rajiv Gandhi Government General Hospital, Chennai. *Corresponding Author

Director , Institute of General Surgery, Madras Medical College and Rajiv Gandhi Government General Hospital, Chennai.

\footnotetext{
Introduction: Fibromatosis is a rare soft tissue tumour that arises from the musculoaponeurotic structures. It usually affect the Abdominal wall but may be also found in other less common sites including head, neck, oral cavity,spinal, limb and sacrococcygeal region. Sacral fibromatosis is one of the rare presentation. We did a wide local excision. Histopathological examination showed that they are composed of fibroblast, fibrocytes and bundles of collagen fibres Case Report: A 32 year old male came with swelling in the sacrococcygeal region. Swelling of size $9 \times 8 \mathrm{~cm}$ in the sacrococcygeal region. FNAC was done, which revealed fibromatosis. MRI was done to look for deeper involvement. Wide local excision was done under anesthesia. Histological examination revealed deep fibromatosis with all resected margins free from tumor

Conclusion: Fibromatosis is a low grade non metastasising tumour, however it tends to recur locally without a complete resection .So long term follow up is recommended.
}

\section{INTRODUCTION}

Fibromatosis commonly develops in the fibrous connective tissue of the body that connects, supports and surrounds other parts and organs. They can develop at any body site. They can be superficial fibromatosis which is less aggressive than deep fibromatosis. The cause of fibromatosis remains unknown . It may be present in any part of the body, it is more common in abdominal wall, it can also occur in head and neck, limb, back, and sacrococcygeal region. Sacral fibromatosis is a very rare presentation. Diagnosis of fibromatosis preoperatively is difficult, MRI can help in diagnosis. However postoperative pathological examinations confirm the diagnosis. Deep fibromatosis is a low grade tumor with no chance of metastasis, it tends to recur locally, so complete resection with margin and long term follow up is required.

\section{Case report}

A 32 year old male came with swelling in the sacral region for the past 2 years, not painful, no limb weakness, no neurological deficit. On examination a swelling of size $9 \times 8 \mathrm{~cm}$ in the sacral region,margins well defined, surface smooth, firm in consistency, skin is pinchable, mobile in horizontal axis, swelling is deep to subcutaneous plane, non tender, no limb weakness, no neurological deficit.

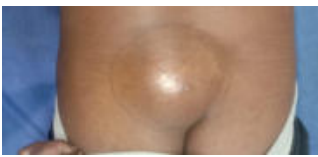

\section{Investigations}

MRI showed $9 \times 9.1 \times 5 \mathrm{~cm}$ lobulated mass lesion noted in the sacral region in the subcutaneous plane

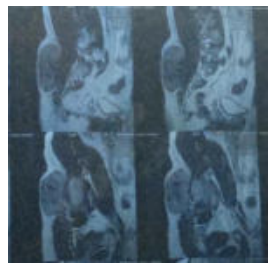

Core Needle biopsy of the lesion showed Fibromatosis Patient was planned for wide local excision under general anesthesia. The swelling was removed in toto with $1 \mathrm{~cm}$ margin all around.

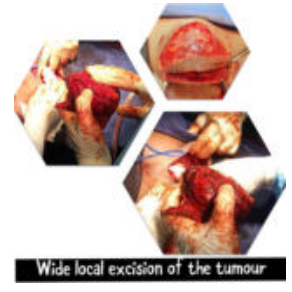

Histopathological examination of the excised tumor showed neoplasm composed of long and short fascicles and sheets of elongated cells with moderate eosinophilic cytoplasm. Mitosis $<1 / 10 H P F$.All the circumferential margin including the posterior resected margin free from tumor. Tumor shows Beta catenin- nuclear positive in $50 \%$ of tumor cells. MSAnegative, S-100 - negative, CD 34-negative, TLE - negative.The diagnosis is deep fibromatosis Post operative patient recovered well. Patient was placed on regular follow up

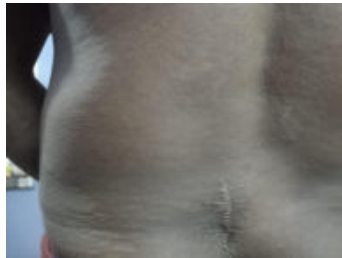

\section{DISCUSSION}

Fibromatosis is a rare soft tissue tumor composed of myofibroblast that arises from the musculoaponeurotic structures.It constitutes $6 \%$ of soft tissue neoplasm and $0.3 \%$ of all neoplasm.

It usually affects the abdominal wall but may be also found in other less common sites including head and neck, limb, oral cavity , spine. It has high potential for recurrence and local invasion, such as muscle and neurovascular structures. The incidence of aggressive Fibromatosis is 2-4 per 10,00,000 per year. It can occur at any age but more commonly occurs between ages of 6 and 15 and again around the age of 40 . The etiology remains unclear, but it may have an association with genetic predisposition, trauma, familial adenomatous polyposis, endocrine factors and Gardner's syndrome. clinical presentation is as symptomatic except for a slowly enlarging tumor. 


\section{CONCLUSION}

Fibromatosis is a low grade non metastatic tumor, however it tends to recur locally without a complete resection. So long term follow up is recommended. 